Questions vives

\section{Questions Vives}

Recherches en éducation

$N^{\circ} 26 \mid 2016$

Questions à la didactique de l'histoire

\title{
"Entre prouesses et petits arrangements » : des professeurs d'histoire-géographie face au changement de prescription
}

"Between prowess and small arrangements": history teachers facing the change of prescription

Brigitte Morand et Guillaume Serres

\section{OpenEdition}

Journals

Édition électronique

URL : http://journals.openedition.org/questionsvives/2005

DOI : 10.4000/questionsvives.2005

ISSN : $1775-433 X$

\section{Éditeur}

Université Aix-Marseille (AMU)

Édition imprimée

Date de publication : 15 décembre 2016

ISBN : 978-2-912643-50-6

ISSN : 1635-4079

Référence électronique

Brigitte Morand et Guillaume Serres, « «Entre prouesses et petits arrangements » : des professeurs d'histoire-géographie face au changement de prescription », Questions Vives [En ligne], № 26 | 2016, mis en ligne le 15 décembre 2016, consulté le 03 mai 2019. URL : http://journals.openedition.org/ questionsvives/2005; DOI : 10.4000/questionsvives.2005

Ce document a été généré automatiquement le 3 mai 2019.

\section{(†)}

Questions Vives est mis à disposition selon les termes de la licence Creative Commons Attribution Pas d'Utilisation Commerciale - Pas de Modification 4.0 International. 


\title{
« Entre prouesses et petits
} arrangements ": des professeurs d'histoire-géographie face au changement de prescription

\author{
"Between prowess and small arrangements": history teachers facing the change \\ of prescription
}

Brigitte Morand et Guillaume Serres

\section{Introduction}

1 Que se passe-t-il, du côté des praticiens de terrain, lorsque les prescriptions imposent à leurs yeux des changements considérables ? Ce fut le cas en 2010 quand les programmes d'histoire-géographie du lycée furent profondément remaniés, tant sur le plan des contenus que des démarches préconisées.

2 Il est courant de pointer, y compris dans les médias qui les dénoncent souvent en termes de corporatisme, les résistances au changement de notre système scolaire. De leur côté, les travaux en didactique ont mis en avant la permanence d'un modèle disciplinaire (Audigier, 1995) au long du XX siècle, malgré les évolutions (épistémologiques ou didactiques) de la discipline. Cherchant « dans le fonctionnement même de la discipline scolaire ce qui en éclaire la stabilité », Nicole Tutiaux-Guillon (2008) décrit la "logique des pratiques disciplinaires du secondaire en histoire-géographie » et propose un modèle qui combine, du côté institutionnel, le découpage du temps scolaire et la logique des programmes et, du côté des enseignants ${ }^{1}$, les conceptions des apprentissages et la logique de l'exposition (du savoir). Elle en conclut que « la solidité entre les composantes stabilise la discipline scolaire $»$.

Partant de ce constat, nous avons voulu savoir comment les acteurs de terrain, partie prenante active de ce système, vivent et composent avec les évolutions de la prescription. 
Nous nous sommes pour cela penchés sur le travail de conception de l'enseignement en amont de la classe, au moment où le professeur, seul, en l'absence des élèves, réalise des choix de contenu et de démarche. Cinq études de cas ont été réalisées auprès de professeurs chevronnés. Elles visent à comprendre ce qui oriente leurs choix et à décrire la façon dont ils les vivent.

Cette recherche a été mise en place pour alimenter des questions de formation. L'idée était de repérer les stratégies mobilisées par des professeurs expérimentés face à ces injonctions de transformation. Les enseignants que nous avons interrogés sont tous de surcroit formateurs de terrain. Ils ne peuvent évidemment pas représenter l'ensemble des enseignants, et les pratiques qu'ils nous ont décrites ne prétendent pas non plus refléter les pratiques majoritaires (Thémines, 2006) dans les classes d'histoire-géographie.

Nous présentons ici les portraits de trois des cinq professeurs ${ }^{2}$ avec lesquels nous avons travaillé. Ils exerçaient tous en classe de première dans des établissements de centre-ville au profil proche. À l'aide du cadre théorique élaboré au cours de notre recherche, ils nous permettent de comprendre comment sont effectués les choix qui conduisent (ou pas) à l'évolution des pratiques de classe. Ces portraits mettent par ailleurs en évidence des façons différentes de se réaliser face à ces nouvelles exigences du métier.

\section{Cadre théorique et méthodologie : La didactique au cœur de l'activité}

6 Cette recherche finalisée par des objectifs de formation nous a conduits à élaborer un cadre conceptuel ad hoc. Une double approche didactique et ergonomique (Robert \& Rogalski, 2002) nous permet d'envisager à la fois ce qui est souhaitable de faire (d'un point de vue didactique) et acceptable (du point de vue de la logique des acteurs). Cette approche est à nos yeux primordiale pour comprendre leurs intentions, multiples, et pour développer une analyse qui rende compte du caractère multi-déterminée et multiadressée de l'activité des enseignants (Goigoux 2007 ; Thémines, 2001, 2005).

7 Nous présentons ci-dessous les principaux points qui structurent notre analyse de l'activité des enseignants lorsqu'ils conçoivent leurs cours. Il s'agit, en s'appuyant sur leurs propres descriptions, de considérer les stratégies, les ruses à l'œuvre (Lantheaume, 2007) ou les opportunités qu'ils saisissent pour faire leur travail, les valeurs et les croyances sous-jacentes, les sentiments qu'ils éprouvent en réalisant ces arbitrages.

Le travail de conception est envisagé comme un effort de lecture, d'interprétation et de redéfinition des prescriptions.

C'est à la tâche redéfinie, au sens de Leplat (1980), que nous nous sommes attachés afin de mettre au jour les tensions propres au travail de conception. Les entretiens réalisés pour le chapitre considéré, avaient pour objectif de permettre aux enseignants de resituer leurs choix. Il s'agissait de comprendre ce qui les conduit parfois à prendre de la distance vis-à-vis de la prescription, vis-à-vis de l'épistémologie de la discipline ou encore vis-à-vis de pratiques qu'ils jugent efficaces et/ou égalitaires, et questionnent leurs valeurs en tant que professeur, en tant que professeur d'histoire géographie, en tant qu'acteur clef de la formation du citoyen.

La dimension didactique est au cour du travail de l'enseignant. 
11 «La principale préoccupation des enseignants (étant de) permettre aux élèves d'apprendre quelque chose", l'analyse didactique est essentielle pour l'analyse de l'enseignement et la compréhension des déterminants et adressages de l'activité (Goigoux, 2007). Par ailleurs, les travaux en didactique de l'histoire et de la géographie ont depuis longtemps montré que l'enseignement de ces disciplines s'inscrit dans un modèle disciplinaire (Audigier, 1995). Ils se sont interrogés sur sa stabilité, mettant en avant un paradigme pédagogique positiviste (Tutiaux-Guillon, 2009) articulant solidairement les contenus, les méthodes et les finalités de ces disciplines. Ainsi s'organise une «logique des pratiques disciplinaires du secondaire en histoiregéographie", un système dans lequel découpage du temps scolaire, logique des programmes, logique de l'exposition (des savoirs), et conceptions de l'apprentissage éclairent cette stabilité, qui peut être perçue comme une résistance au changement (Tutiaux-Guillon, 2004). Ce modèle revient donc à faire des enseignants à la fois des sujets actifs, mais aussi finalement captifs de ce système. Dans le contexte de changement de la prescription décrit plus tôt, l'enjeu de notre recherche en didactique réside dans l'exploration du « champ du possible des acteurs »(Doussot \& Thémines, 2016) 3 . Il s'agit dès lors de mieux comprendre comment ces derniers renormalisent chacun à leurs façons leur activité ceci en prenant la mesure du poids des déterminants et des jeux d'adressage qui lui sont inhérents.

Accéder aux choix des acteurs sur la construction du cours

13 Notre étude a été menée sur la base d'entretiens individuels d'environ trois heures réalisés entre les deux chercheurs et les enseignants. Ils avaient arrêté leurs choix et réalisé pour tout ou partie le programme relatif à la guerre froide. Il s'agissait de les remettre en situation (Theureau, 2010) afin qu'ils décrivent, de façon située, la façon dont ils avaient composé avec ces nouvelles prescriptions.

14 Notre but n'était pas de juger du bien fondé des choix des enseignants, ce qui a été clairement posé à chaque début d'entretien. Il était important d'éviter la position de «surplomb» des chercheurs que les professeurs auraient pu ressentir, et de leur donner plutôt « le rôle de l'informateur de l'ethnographe » (Doussot, 2014). La consigne donnée en début d'entretien était de s'adresser directement et prioritairement au chercheur non spécialiste de la discipline et de son enseignement. Nous avons également expliqué que nous cherchions à comprendre pour quelles «bonnes raisons" (Charlot, 1997, cité par Broustail \& Sieper, 2005) les enseignants choisissent de privilégier tel contenu, telle démarche, tel type de document ou support, telle partition entre les activités réalisées en classe avec le guidage du professeur et les activités dévolues aux élèves seuls, hors classe.

Les entretiens se sont déroulés selon un scénario identique. Deux tâches successives ont été demandées : 1) Placer sur un schéma formé de cercles concentriques les mots-clés (notions, concepts, démarches, questionnements) qui ont présidé à la conception de leur enseignement, en distinguant le plus central du plus périphérique. Et ceci sur un temps restreint (vingt minutes) ; 2) décrire plus longuement et pas à pas leur démarche, en s'appuyant sur leurs fiches de préparation et les documents et supports utilisés dans leurs classes. Et ce faisant, d'expliquer les raisons de leur choix.

16 Les cas ont été constitués de façon à comprendre les permanences et les recompositions dans un contexte d'évolution de la prescription. L'analyse ne cherche pas à produire de la généralité mais plutôt à décrire et apprécier divers arbitrages ainsi que la façon dont les enseignants les vivent. La comparaison de ces études de cas permet de percevoir les 
points communs mais aussi les nuances dans leur rapport à la prescription, au métier et à eux-mêmes.

\section{Un programme, trois professeurs, trois démarches}

\subsection{Le programme de première de 2010 : une injonction à l'innovation}

Probablement plus encore que pour les autres disciplines, l'enseignement de l'histoire dans le secondaire est en tension entre les différentes logiques qui concourent à la l'élaboration des prescriptions: logique disciplinaire (évolutions épistémologiques), logique de la forme disciplinaire aux prises avec les évolutions didactiques et pédagogiques, logiques politiques et idéologiques (liées aux finalités civiques traditionnellement attribuées à l'histoire), voire médiatiques (chaque nouveau programme étant l'occasion, pour les tenants de ces différents pôles, de faire valoir parfois de façon virulente leurs arguments) ${ }^{4}$. Le programme de première de 2010 n'a pas échappé à la règle, d'autant plus que les changements qu'il préconise touchent toutes ces logiques en même temps. Très critiqué à sa sortie parce qu'il s'inscrivait aussi dans le cadre de la suppression de l'enseignement de l'histoire en terminale scientifique, il fut aussi qualifié de «monstre pédagogique » par le collectif « aggiornamento », créé par des enseignants déclarant refuser de le mettre en œuvre.

Notre étude porte sur la partie du programme qui concerne la guerre froide, un chapitre qui connaît, outre le glissement de la terminale vers la première, des bouleversements importants dans les contenus et dans les méthodes d'enseignement préconisées. La comparaison des programmes de 2002 et 2010 permet d'en mesurer la portée. La figure 1 présente les plans de séquence tels qu'ils ressortent des programmes, mais aussi des documents d'accompagnement (programme 2002) et des fiches ressources pour la classe (programme 2010). 
Figure 1 : Enseigner la guerre froide d'après les Instructions Officielles de 2002 et 2010

\begin{tabular}{|l|l|l|}
\hline & \multicolumn{1}{|c|}{ Programme terminale 2002 ${ }^{1}$} & Programme première 2010 \\
\hline $\begin{array}{l}\text { Partie du } \\
\text { programme }\end{array}$ & Le monde de 1945 à nos jours & La guerre au XXº siècle \\
\hline $\begin{array}{l}\text { Intitulé du } \\
\text { chapitre } \\
\text { guerre froide }\end{array}$ & $\begin{array}{l}\text { - Les grand modèles et la } \\
\text { confrontation Est-Ouest jusqu'aux } \\
\text { années 70 } \\
\text { - Le Tiers-Monde : indépendances, } \\
\text { contestations de l'ordre mondial, } \\
\text { diversification }\end{array}$ & $\begin{array}{l}\text { La guerre froide, conflit } \\
\text { idéologique, conflit de } \\
\text { puissances : } \\
\text { un lieu (Berlin 1945-1989), } \\
\text { une crise (Cuba 1962), } \\
\text { un conflit armé (la guerre du } \\
\text { Vietnam) }\end{array}$ \\
\hline $\begin{array}{l}\text { Organisation } \\
\text { de la } \\
\text { séquence }\end{array}$ & $\begin{array}{l}\text { 1. « Lignes de force » de la politique } \\
\text { internationale de 1945 à 1970 } \\
\text { - De 1945 à 1963 : la guerre froide } \\
\text { - De 1963 à 1970 : la détente }\end{array}$ & $\begin{array}{l}\text { 1. Berlin (1945-1989) } \\
\text { 2. La crise de Cuba (62) } \\
\text { 3. La guerre du Vietnam (64- } \\
\text { 75) } \\
\text { 2. Modèles idéologiques américains } \\
\text { et soviétiques } \\
\text { 4. La guerre froide : } \\
\text { conflit idéologique, conflit de } \\
\text { puissance }\end{array}$ \\
$\begin{array}{l}\text { 3. Le Tiers-Monde (...) } \\
\text { 4. 1970-nos jours : A la recherche } \\
\text { d'un nouvel ordre mondial. Retour } \\
\text { des tensions et fin de la Froide }\end{array}$ & \\
\hline $\begin{array}{l}\text { Temps } \\
\text { consacré à la } \\
\text { guerre froide }\end{array}$ & $\begin{array}{l}\text { 8̀ 10 h } \\
\text { 4h }\end{array}$ & \\
\hline
\end{tabular}

Dans le programme de 2002, le récit commence par les causes de la guerre froide (la fin de l'alliance entre "grands » et la mise en place des blocs antagonistes), pour ensuite dérouler le fil des événements organisés selon la périodisation traditionnelle (découpage en phases selon l'intensité du conflit : « la détente », « la guerre fraîche »). Le Tiers Monde (que l'on reprenait en 1945) s'intercalait, dans une séquence qui tenait une place horaire importante (environ $1 \frac{1}{4}$ du temps imparti à l'histoire).

Dans le programme de 2010, le récit chronologique est rompu: il s'agit désormais de partir de l'étude d'un événement particulier (Crise de Cuba, du Vietnam) ou d'un lieu emblématique du conflit (Berlin) à partir du duquel, en généralisant ensuite à l'espace mondial, on débouche sur la caractérisation du conflit Ouest-Est : « un conflit idéologique, un conflit de puissances ».

21 Cet abandon du récit chronologique représente une remise en cause fondamentale $d u$ modèle disciplinaire qui prévalait encore jusque-là (malgré d'importantes évolutions) dans l'organisation des programmes (Audigier, 1995). Élaboré au XIXe siècle par ceux qui furent aussi les fondateurs de l'histoire comme discipline scientifique, ce modèle reposait sur une conception de l'histoire en tant que science, sur les finalités assignées à son enseignement, sur une conception du rôle du professeur (et donc des élèves) dans sa mise en œuvre.

22 - Sur le plan épistémologique, le récit chronologique permettait de donner une vision progressiste de l'histoire de l'humanité (progrès des sciences, des techniques, de la démocratie...) tout en proposant, implicitement, une interprétation de cette histoire : les événements s'organisent en une ligne continue de causes et de conséquences (Lautier, 2003). Le travail de l'historien (et donc ensuite du professeur) consiste alors à élucider ces liens de causalité. 
23 nationale qui, au fil des événements et de l'action des "grands hommes », aboutissait à l'avènement inéluctable de la République et de la société française contemporaine (Audigier, 1995). Si aujourd'hui le « roman national» n'a heureusement plus sa place tel quel, il a encore ses défenseurs, et les programmes restent cependant largement structurés autour de l'histoire nationale (Legris, 2010, 2014). De même, si la continuité chronologique stricte est abandonnée depuis longtemps ${ }^{5}$, la linéarité est encore le principe d'organisation générale. Or c'est à ce principe que s'attaque le programme de première de 2010, en imposant des allers-retours dans le temps qui bousculent l'ordre habituel du traitement de certains thèmes ${ }^{6}$. L'objectif affiché est l'enseignement d'une histoire moins "encyclopédique", plus conceptuelle et plus problématisée. Ainsi la guerre froide n'est plus enseignée comme une période « à part » mais intervient dans une réflexion globale sur « la guerre au XX⿳亠丷厂犬 siècle » (BOEN spécial n 9, 30 septembre 2010).

24 faisant reposer l'essentiel des apprentissages sur le travail autonome de l'élève à partir d'un dossier documentaire, bouscule les conceptions de l'enseignement-apprentissage encore largement partagées par les enseignants d'histoire-géographie. (Tutiaux-Guillon 2004). Ces thèmes d'étude bouleversent également les habitudes, en demandant de travailler à partir d'exemples précis dans une démarche qui s'apparente à celle des études de cas de la géographie. Toutefois, si pour la géographie les Instructions officielles parlent clairement de démarche inductive, la fiche « Démarches pour la mise en œuvre du programme d'histoire » est moins explicite ${ }^{7}$.

La mise en œuvre du programme de première de 2010 a donc offert une opportunité intéressante pour observer la façon dont les professeurs composent avec les nouvelles prescriptions. Les entretiens réalisés nous permettent de documenter les choix de découpage des contenus et de leur mise en ordre (maintien d'une continuité chronologique, démarche par étude de cas, compromis entre les deux approches), les ressources mobilisées (frises chronologiques, cartes, textes, images fixes ou animées) les supports et de leurs usages (power point, tableau interactif...), les mises en œuvre (cours magistral dialogué, étude de cas en autonomie sur dossiers de documents, mise en activité ponctuelle des élèves sur documents), tout comme les choix de partition des tâches entre activité en classe et hors classe.

\subsection{Trois « portraits didactiques »}

La figure 2 montre que les séquences qui nous ont été présentées ne suivent plus l'ordre chronologique. Ce trait commun ne masque cependant pas des façons différentes de composer avec cette prescription.

27 C'est dans l'organisation des séquences que les différences de choix paraissent au premier abord les plus nettes, même si elles méritent d'être examinées de plus près. La figure 2 met en évidence deux types de stratégies :

- Monique et Jérôme se montrent très proches de la démarche prescrite en entamant la séquence directement avec l'étude de Berlin : "J'ai estimé que... il fallait aborder d'abord Berlin de 48/49 ", commente Jérôme, et ceci « avant tout baratin » nous dit Monique (fig. 3).

- Tandis que Madeleine s'en écarte nettement en choisissant de commencer par une séance de 2 heures qui, à l'aide d'une carte situant les blocs en présence et les principaux événements, lui permet de poser son «cadre » contextuel et conceptuel.

Questions Vives, $N^{\circ} 26$ | 2016 
Figure 2 : Trois plans de séquences

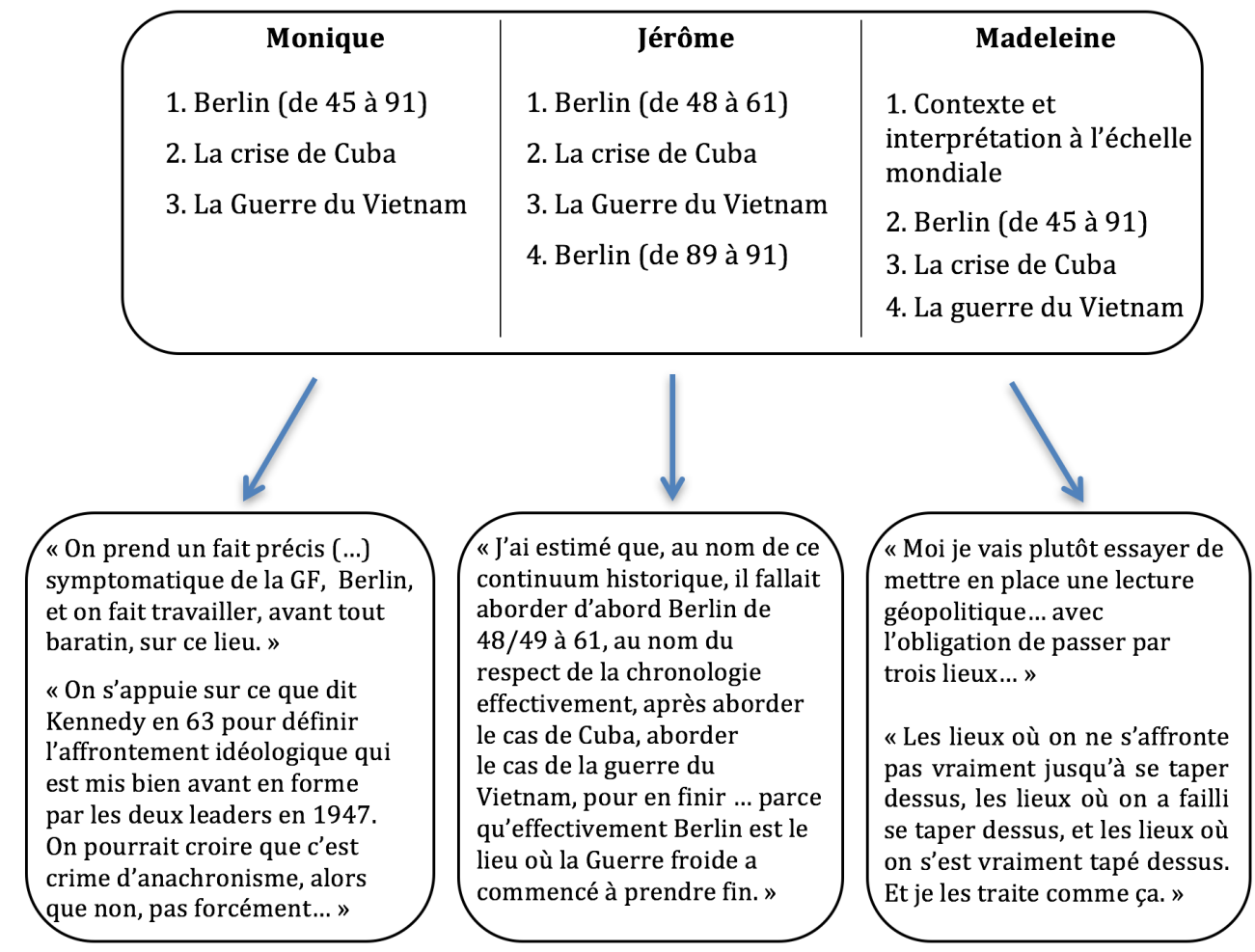

28 La figure 3 révèle cependant un schéma plus complexe : si, au contraire de Madeleine, les plans des séquences décrits par Jérôme et Monique respectent la démarche préconisée (fig. 3, Monique: «Etude de cas, mise en perspective, élargissement »), on constate chez ces deux professeurs des mises en œuvre bien différentes :

- Jérôme, qui conduit lui-même l'étude, utilise de façon quasi exclusive le "cours interactif»" selon son expression (c'est-à-dire le cours magistral dialogué),

- tandis que Monique a recours (certes ponctuellement voire en externalisant) à la «mise en activité» des élèves sur des documents,

- ce qui la rapproche cette fois de Madeleine chez qui (à l'exception notable de la séance de cadrage du début), les séances sont entièrement basées sur l'activité des élèves sur documents (fig. $3:$ : ils travaillent sur les documents, et on fait après un corrigé rédigé »).

Que peut-on percevoir des raisons de ces choix au travers des propos tenus dans les entretiens? 
Figure 3 : Trois démarches

\begin{tabular}{|c|c|c|}
\hline Monique & Jérôme & Madeleine \\
\hline $\begin{array}{l}\text { Pour chaque étude : } \\
\text { 1. Contexte (très rapide - } \\
\text { cours dialogué) } \\
\text { 2. Activité sur documents } \\
\text { 3. Correction (dialogue) et } \\
\text { trace écrite } \\
\text { 4. Elargissement (cours } \\
\text { dialogué) }\end{array}$ & $\begin{array}{l}\text { Pour chaque étude : } \\
\text { 1. Contexte (très rapide - } \\
\text { cours dialogué) } \\
\text { 2. Cours dialogué avec } \\
\text { appui sur documents } \\
\text { 3. Elargissement } \\
\text { (dialogue) }\end{array}$ & $\begin{array}{l}\text { Après contextualisation et } \\
\text { interprétation ( } 2 h \text { ) } \\
\\
\text { Pour chaque « exemple »: } \\
\text { 1. Activité en autonomie } \\
\text { sur documents (avec fiche } \\
\text { de travail) } \\
\text { 2. Correction (dialogue) et } \\
\text { trace écrite }\end{array}$ \\
\hline $\begin{array}{l}\text { "C'est la même démarche } \\
\text { en géo. Etude de cas, mise } \\
\text { en perspective, } \\
\text { élargissement. " } \\
\text { "Avant toute autre forme } \\
\text { de procédé on contextualise } \\
\text { un peu, on dit voilà, on va } \\
\text { faire ça pour ça, on étudie } \\
\text { ça parce que ça... » } \\
\text { "On attaque par une mise } \\
\text { en activité. Et ensuite on va } \\
\text { bâtir le cours, à partir des } \\
\text { réponses. Et après, le travail } \\
\text { du prof, le job du prof, c'est } \\
\text { de généraliser à partir de } \\
\text { l'exemple. » }\end{array}$ & $\begin{array}{l}\begin{array}{l}\text { "C'est une étude qui doit se } \\
\text { suffire à elle-même et qui } \\
\text { doit déboucher sur quelque } \\
\text { chose de plus globalisant." } \\
\text { "J'ai souvent tendance à } \\
\text { débuter mes séquences par } \\
\text { une frise chronologique } \\
\text { pour situer dans le temps } \\
\text { les événements que l'on va } \\
\text { aborder ... » } \\
\text { "Pour les choses qui me } \\
\text { semblent assez évidentes, je } \\
\text { ne donne pas de fiche, pas } \\
\text { de travail en autonomie, } \\
\text { c'est le cours interactif... " } \\
\text { "J'essaie vraiment de } \\
\text { grossir la focale pour } \\
\text { passer de mon cas précis à } \\
\text { une vérité peut-être plus } \\
\text { générale, plus globale. » }\end{array}\end{array}$ & $\begin{array}{l}\text { "En fait c'est du cours } \\
\text { magistral, là. On regarde } \\
\text { les cartes, on localise... » } \\
\text { "Alors après c'est les } \\
\text { exemples... » } \\
\text { "Je leur présente la carte et } \\
\text { j'explique la situation sur la } \\
\text { carte. Et puis après ils } \\
\text { travaillent sur les docs, et } \\
\text { on fait après un corrigé } \\
\text { rédigé... Je leur donne les } \\
\text { docs à l'avance pour qu'ils } \\
\text { les débroussaillent un petit } \\
\text { peu... » }\end{array}$ \\
\hline
\end{tabular}

\section{Des choix didactiques en accord avec les conceptions du métier de professeur d'histoire- géographie}

30 L'analyse de ces trois portraits permet de poser les bases de ce qui sera l'objet d'une étude à plus vaste échelle. On peut déjà en tirer quelques éléments, tant sur le plan didactique que sur la question du rapport à la prescription et, plus largement, du rapport au métier (Fontanabona \& Thémines, 2005).

\subsection{Des choix qui témoignent d'une évolution du rapport à la discipline}

31 On peut en effet constater des évolutions dans la conception des cours (fin de la continuité chronologique, approche plus conceptuelle et problématisée du savoir), évolutions qui témoignent d'une reconfiguration du modèle disciplinaire du moins dans ce qui touche au rapport au savoir (épistémologie).

Fin de la continuité chronologique

33 Malgré des choix de scénario très différents, les séquences qui nous ont été présentées ne suivent plus l'ordre chronologique. Cette rupture avec le modèle disciplinaire est d'autant plus remarquable qu'elle concerne un chapitre où la continuité chronologique (comme d'ailleurs pour tous les chapitres à forte densité "événementielle», la Révolution française par exemple) est difficile à abandonner puisque la causalité linéaire 
( l'engrenage des événements ) a constitué pendant longtemps le mode d'explication implicite de l'histoire. Il est aussi difficile de se détacher d'une conception qui veut que l'on apprenne d'abord «les bases ", c'est-à-dire des repères chronologiques, avant toute réflexion conceptuelle (jusqu'ici les programmes du collège étaient d'ailleurs explicitement construits sur ce postulat). Cet abandon de la chronologie ne va cependant pas sans interrogations, et suppose même, pour au moins deux d'entre eux, d'importants renoncements.

Ainsi Jérôme a du mal à renoncer totalement à une démarche dans laquelle il a été formé :

"Moi qui avais connu l'enseignement avant, où on faisait "cause, manifestations, conséquences", ben c'est plus ça.» Il a donc trouvé un compromis, ce qui l'a conduit "à prendre un peu de distance avec les attentes de l'institution. » Il coupe donc en deux l'étude de Berlin pour, " au nom du continuum historique " y insérer celle sur la crise de Cuba.

Sa conception de l'histoire est donc clairement engagée dans ce choix, cohérent avec ce qu'il considère comme la méthode la plus profitable pour la compréhension des élèves : "Je suis revenu à une méthode qui m'a paru simple, mais qui peut être simpliste pour d'autres, où il y une suite chronologique, et l'élève a une sorte d'axe de la pensée où il voit les événements se succéder, où il y a une certaine forme de logique, pas de déterminisme, mais de logique."

Pour Madeleine ce choix est encore plus coûteux, puisqu'elle juge réductrice et finaliste ("téléologique») l'histoire qu'on lui demande d'enseigner. Pourtant, elle «assume complètement cette dérive intellectuelle, fâcheuse sur le plan scientifique (...). Je fais une histoire complètement déformée, à usage de ce que je veux montrer par la suite... Et la commande étant Berlin, le Vietnam, Cuba, je passe au tamis et j'enlève le reste."

Monique semble la plus convaincue des trois: "Je trouve cela intellectuellement très séduisant» et fait le choix de "travailler autrement que dans le récit». Les raisons qu'elle évoque avec enthousiasme montrent une adhésion aux évolutions de la discipline, tant sur le plan idéologique qu'épistémologique: "Le récit linéaire... c'était presque une guerre froide roman national. Mais aujourd'hui il y a eu autre chose après la guerre froide qui la met en perspective et je crois qu'on nous demande d'aller à l'essentiel et d'enlever les scories. »

\section{Enseignement d'une histoire plus conceptuelle, plus problématisée}

39 Fiche ressource eduscol à l'appui ${ }^{8}$, Monique nous en explique les objectifs : «enseigner la guerre froide, c'est enseigner la guerre», ce qui justifie l'abandon des phases de la périodisation traditionnelle: "Aujourd'hui on va à l'essentiel, on problématise. On ne va plus entrer dans le détail pour savoir si on est dans une guerre froide intense, moyennement intense puis plus du tout intense... car cela n'a pas beaucoup de sens pour ce qu'on veut leur faire comprendre. ». L'essentiel est donc pour elle de "faire comprendre », ce qui explique son choix de commencer chaque étude par une courte phase de contextualisation et de problématisation: "on dit voilà, on va faire ça pour ça, on étudie ça parce que ça. Ils comprennent, les élèves, si on leur dit. »

Madeleine a adopté la même démarche, mais consacre beaucoup plus de temps (deux heures en début de séquence) à la présentation du « cadre ». Cette séance, qui n'est pas organisée chronologiquement, a pour objectif de donner aux élèves une vision générale de la question, là aussi en proposant un sens, une interprétation : "On a développé tout ce qui a trait aux idéologies, on a expliqué tout ce qui était stratégie, les lieux d'affrontement militaire, et les lieux d'affrontement idéologique, ce qu'on appellerait le " soft power » en quelque sorte. ». Les études qui suivent servent ensuite à illustrer cette lecture: «(Berlin) est l'exemple de la guerre froide au sens strict ». 
41 Monique et Madeleine construisent donc leur séquence autour d'une interprétation des événements et de la période, même si les choix de problématique sont différents: Monique insiste plutôt sur les aspects idéologiques et la notion de propagande : "C'est important, ça figure dans les prescriptions ministérielles, il importe de bien la faire apparaître. ", tandis que Madeleine choisit plutôt une «lecture géopolitique» (fig. 2). Cette « lecture » de l'histoire ne s'affranchit évidemment pas totalement de la chronologie et tous trois utilisent une frise en début de séquence afin de présenter l'ensemble de la période. Toutefois cette utilisation ne poursuit pas le même but : si pour Monique et Madeleine la frise sert d'appui à la conceptualisation, pour Jérôme, elle vise surtout à introduire la séance en faisant appel aux souvenirs que les élèves peuvent avoir gardé de la classe de troisième : "L'objectif étant que l'un d'entre eux nous dise "Monsieur, c'est la guerre froide » et qu'on puisse déjà commencer à débroussailler un petit peu l'acception de cette appellation "guerre froide »." Il y consacre d'ailleurs peu de temps : "C'est vraiment très rapide, on recadre, on essaie de ne pas trop laisser de champ libre à des questions. ".

On remarque donc logiquement, chez Monique comme chez Madeleine, un lien entre le choix d'une histoire plus conceptuelle et plus problématisée et l'abandon (certes plus ou moins facile) de la chronologie. Jérôme par contre met beaucoup moins en avant la conceptualisation, et c'est celui qui se montre le plus attaché à la chronologie.

\subsection{Des choix qui permettent de rester en accord avec sa conception du métier} lecture de la prescription et cite avec précision la fiche ressource ${ }^{9}$. Jérôme et Monique discutent également de cette "démarche inductive» (« une étude qui doit se suffire à elle-même et qui doit déboucher sur quelque chose de plus globalisant ", Jérôme, fig. 2) et évoquent la différence entre les études de cas en géographie et les thèmes d'étude en histoire, même si Monique ne s'embarrasse pas de ces subtilités : «On nous demande de travailler avec des études de cas en géographie, et en histoire avec des études ciblées. C'est la même chose ». Madeleine par contre ne semble pas préoccupée par ce problème : pour elle ces thèmes d'étude sont simplement « des exemples » qui viennent illustrer les notions abordées dans la séance de cadrage introductive (fig. 3).

les faits, c'est sur la place accordée au travail des élèves en autonomie que les choix divergent le plus. L'enjeu associé est la question de la place et du rôle de la parole professeur dans la classe. Chez Jérôme, l'activité des élèves tout au long de la séquence consiste principalement à répondre oralement aux questions du professeur : «Pour des choses qui me semblent assez évidentes je ne donne pas de fiche, et pas de travail en autonomie, c'est-à-dire que c'est le cours interactif, là encore c'est pour gagner du temps. Parce que l'interactivité, question/réponse, crac crac, c'est rapide, hop, c'est évacué. »

L'introduction de la nouvelle démarche a donc bien conduit Jérôme à modifier l'ordre d'exposition du savoir par le professeur (il procède bien par thèmes d'étude), mais cela elle ne semble cependant pas déboucher sur une recomposition de la forme du cours (magistral dialogué). Tandis que Monique laisse davantage de place à l'activité des élèves (fig. 3), quoique soigneusement minutée : « ça dure un quart d'heure. Il ne faut pas que ça dure plus, sinon on n'a pas le temps de faire la classe, d'avancer le cours ». Enfin, chez 
Madeleine la séance de cadrage se déroule selon la forme classique du cours magistral dialogué ("théâtralisé », dit-elle), mais l'étude des "exemples» se fait à partir d'un travail sur document, largement réalisé en autonomie, selon une démarche semble-t-il assez proche de celle de Monique (fig. 3).

On ne voit donc pas ici de corrélation entre l'adoption de la démarche inductive par thèmes d'étude et la transformation de la forme du cours. Il semble plutôt que ces enseignants aient composé, chacun à leur manière, avec leur méthode habituelle, qui correspond probablement à leurs conceptions de l'enseignement-apprentissage (TutiauxGuillon, 2004). Ces dernières ne sont cependant pas clairement exprimées (sauf chez Madeleine) et ressortent plutôt en filigrane de leurs discours (Fontanabona \& Thémines, 2005). On peut le voir chez Monique, pour qui faire travailler les élèves sur les documents entre en compétition avec "faire la classe", ce qui suppose une conception de l'enseignement-apprentissage reposant principalement sur la parole du professeur. On le voit aussi chez Jérôme, pour qui le rôle des élèves est de «suivre le cours »: « Si vous suivez mon cours je pense que vous aurez les bases notionnelles pour faire une bonne dissertation et une bonne étude de documents en histoire ». Madeleine est la seule des trois à évoquer clairement ses conceptions, parce que son choix de scénario les met en tension. Elle doute ainsi beaucoup de sa séance de cadrage, qui introduit des notions que les élèves n'ont encore pas travaillées : «Je me suis toujours demandé si je faisais bien de leur donner un exemple que je n'allais leur montrer qu'après... Ils sont obligés au départ de me croire sur parole, parce qu'ils n'ont pas encore vu le film sur Berlin, donc c'est peut-être contestable.» On perçoit aussi son peu de goût pour la parole d'autorité, dont elle doute de l'efficacité : « Historiquement, mais là c'est un peu ma culture personnelle, je peux faire du récit, momentanément, mais je ne suis pas adepte du cours comme ça, ex cathedra. Il me semble que si les élèves ne s'approprient pas, par une activité quelconque, ce qu'on est en train de leur dire, c'est un peu du temps perdu (...) Il y a des élèves pour qui rester à l'écoute c'est très difficile. ». La contrainte du temps imparti et le bon niveau de ses élèves (qui «acceptent assez facilement un récit du professeur») l'ont donc conduite à des choix qui ne la satisfont pas et elle exprime ses doutes à plusieurs reprises.

Ce maintien du rôle traditionnel du professeur est aussi conforté par une contrainte forte imposée par le programme, celle du temps. La contrainte du temps peut d'abord présider au choix des documents, comme Jérôme et Madeleine qui commencent (chacun à la leur façon) leur séquence par l'étude de cartes à leurs yeux plus faciles d'accès pour les élèves que les textes, surtout à certaines heures de la journée : «J'aime bien partir de cartes. Là aussi l'idée c'est d'aller vite, là j'avoue c'est pour gagner du temps. Les élèves en plus des fois je les avais de $16 \mathrm{~h}$ à $17 \mathrm{~h}$ après $2 \mathrm{~h}$ de math, ils étaient cuits. Des fois c'était un confort pour moi d'avoir des trucs assez évidents à leur faire entrevoir et pour moi ça permettait de gagner du temps.» (Jérôme). "Je ne vois pas comment on peut faire, dans le peu de temps qu'on a, si on n'aborde pas la question avec une carte. » (Madeleine).

Le « temps imparti » influence aussi le choix des démarches, comme pour Madeleine qui «théâtralise » sa séance de cadrage « pour que cela tienne » ou pour Monique qui limite le travail sur documents dans la classe, voire qui externalise : «Ça peut être aussi donné en préparation d'une fois pour l'autre (...). Je joue sur les 4-5 heures quand je demande faire les exercices à la maison. Ce qui n'est pas la prescription. » Madeleine a également recours au travail préparé à la maison: "Je leur donne les documents à l'avance pour qu'ils les débroussaillent un petit peu " (fig. 3), de même que Jérôme, ce qui pour lui consiste à « regarder » à la maison, » lorsque le document est important » : «Je l'ai donné 
à regarder à la maison, parce que c'est un truc assez long, parce que ça fait appel à certaines connaissances et j'avais l'envie que les élèves comprennent pourquoi ce mur a été construit. ». Monique joue même sur toutes les possibilités qui sont à disposition pour gérer cette contrainte : «Il faut y passer $4 \mathrm{~h}$. Ça oblige à réfléchir autrement sinon on est mort. » Elle utilise aussi les heures d'accompagnement personnalisé pour «avancer le cours " lorsque l'exercice s'y prête bien, comme pour le travail à partir du site du mémorial de Caen : «J'ai des heures d'accompagnement personnalisé où je peux faire de la méthodologie propre à l'histoire-géographie, par exemple, et où je peux faire: " comment on extrait de l'information, non pas d'un doc qui est dans le livre, mais d'un site.» Monique utilise donc tous les moyens mis à disposition par la prescription : non seulement elle décide de consacrer une heure de plus à la guerre froide (quitte à réduire d'autant un autre chapitre) "parce qu'il y a du mou", mais elle investit aussi les nouveaux espaces pédagogiques (l'accompagnement personnalisé). Ces trois enseignants pratiquent donc l'externalisation du travail des élèves, pourtant aucun d'eux n'en est totalement satisfait.

Toutefois, quels que soient les choix de démarches, dans les trois cas la tâche de généralisation reste à la charge du professeur : aux élèves le travail sur documents pour découvrir les événements et les enjeux (ce qu'ils peuvent d'ailleurs faire chez eux, pour gagner du temps), à l'enseignant la mise en perspective : soit en amont du travail des élèves (comme chez Monique, fig. 3 : « on va faire ça pour ça », ou chez Madeleine avec sa séance de " cadre »), soit en aval (Monique, fig. $3:$ «le job du prof, c'est de généraliser »). Cette pratique est décrite également par Doussot (2014) chez une enseignante du primaire, réticente au départ à laisser la tâche de généralisation à ses élèves, ainsi que par Boutonnet (2015) à propos cette fois d'enseignants québécois chez qui « la structuration de l'interprétation historique est contrôlée par l'enseignant puisque tout est choisi (...) afin de dévoiler le savoir au fur et à mesure ». Cette pratique est d'ailleurs confortée par la prescription elle-même : si les thèmes d'études insistent sur la nécessité du travail sur document, celui-ci «n'est pas un objet pédagogique verrouillé » mais « un instrument dans la panoplie du professeur », précise la fiche-ressource Eduscol ${ }^{10}$.

Le rapport à la prescription est très fort chez ces trois enseignants, il est sans surprise une dimension fondamentale de leur perception du métier. Et lorsqu'ils choisissent de s'en écarter, ce sont pour de «bonnes raisons » qu'ils tiennent tous à souligner. C'est le cas de Jérôme, à propos de son choix de couper en deux l'étude de Berlin : »C'est un choix personnel, qui prêterait peut-être le flan à la critique d'une manière justifiée (...) J'ai pris sur moi, en biaisant un peu ou en mettant à ma sauce, si j'ose dire, des prescriptions, qui à mon sens demandaient à étudier ces événements en eux-mêmes, et donc de traiter Berlin en un bloc». Cette "prise de distance avec les attentes de l'institution» lui pose problème, si l'on en croit ce qu'il dit à propos de ses choix lorsqu'ils sont en adéquation avec cette attente : "La crise de Cuba comme la guerre du Vietnam, il n'y a pas vraiment de cas de conscience, à mon sens, à se poser ». À l'opposé, Madeleine déplore que la fin de la continuité chronologique conduise à enseigner "une histoire déformée ", cependant elle « assume tout à fait » cette démarche, dans le respect de la prescription pour l'intérêt des élèves.

Ce rapport à la prescription peut aussi s'exprimer de façon plus positive, et même revendiqué lorsqu'il correspond à des valeurs du métier. Ainsi pour Monique, l'intérêt pour la démarche par thèmes d'études se double d'une conviction qui la renforce dans sa 
volonté d'être au plus près de la prescription : «On pourrait rétorquer «c'est la voix de son maître ", c'est vrai. C'est la voix du ministère. Et heureusement qu'il y en a une, pour homogénéiser les pratiques un tant soit peu, et faire en sorte que les élèves, qui vont être évalués au bac là-dessus, aient des cours où il y a une liberté pédagogique indéniable, mais qui soient quand même balisés par cette affaire. Parce que sans ce balisage-là, on va vers une iniquité considérable. » Pour Monique, une forte homogénéité des pratiques est donc une garantie d'équité pour les élèves.

Pour Jérôme, le souci de la réussite de ses élèves au baccalauréat semble la raison d'être de son activité : «Je pars toujours de la demande, avec cette idée de coller au plus près 1) à ce qu'on me demande et 2) à ce qu'on pourrait demander à mes élèves au bac. ». Il y revient plusieurs fois au cours de l'entretien: «Quand on a une première littéraire ou économique et sociale, que le programme soit terminé ou qu'il ne soit pas terminé, même si c'est pas bien, c'est pas péjorant pour l'élève parce qu'il n'a pas à répondre finalement de ce qu'il a appris au cours de l'année au bac, qu'il ne passera qu'en terminale. En première scientifique, il n'y a pas le choix : il faut que mordicus, début juin, les élèves y aient fini le programme et d'histoire et de géo... » Et si l'élève doit « répondre » de ce qu'il a appris, le professeur est là pour lui fournir les «bases notionnelles nécessaires et suffisantes » : « Moi ça a toujours été mon leitmotiv, de leur dire : ce que je vous donne ça me paraît nécessaire et suffisant pour être crédible au bac. »

Chez Madeleine, les choix sont également « déterminés » (selon ses propres termes) par la prise en compte des attentes à l'examen, plus précisément les attentes des correcteurs, ce qui la pousse à conserver, dans son cadrage introductif, une part de chronologie : «Je connais la demande (...) je connais aussi la demande des examens (...), il y a un déterminant local clairement. Les correcteurs de l'académie son redoutables. Ils ont des exigences à mon avis plus encyclopédiques que celles du programme. J'essaie d'en tenir compte. »Ce compromis semble aussi correspondre à sa conception, selon ses propres mots, de sa « mission » : «Qu'est-ce qui reste dans le monde aujourd'hui de cette période dont les élèves aient besoin au quotidien? Moi je vise surtout, ça c'est mon expérience de parent qui m'oriente un peu, je vois bien qu'il y a des gens qui ont beaucoup de mal à s'orienter dans la chronologie, dans l'histoire, pour qui le temps est une vrai difficulté (... ). Je me dis qu'il faut quand même donner un peu de sens au monde dans lequel on est, donc simplement j'essaie de poser des jalons, des repères qui vont être faciles à s'approprier, avec des choses un peu caricaturales, voilà, les Américains sont très malheureux mais Reagan arrive sur son cheval, des choses comme ça. »

Façons de se réaliser

Chacun à sa façon ${ }^{11}$ semble donc trouver dans ses choix des "façons de se réaliser ». Madeleine semble trouver un sens dans le sentiment de répondre aux besoins de repères des élèves. Elle y trouve la satisfaction de la «mission accomplie » lorsqu'elle constate que les élèves ont retenus ces repères : «Et ça je sais que ça a marqué parce que quand je retrouve des élèves l'année suivante ils me ressortent la formule "America is back". Pour moi c'est mission accomplie. Ça veut dire ça y est, ils ont un ancrage, cette année-là, et ça reste. ». Cette " mission » s'élargit même au-delà de la classe, puisque dans ses propos elle évoque «les gens » qui ont du mal à s'orienter dans le temps. Madeleine semble donc animée par la finalité civique, d'éducation du citoyen, dévolue à l'enseignement de l'histoire (et de la géographie). Ayant une formation de géographe, elle se réalise dans la façon d'introduire cet enseignement avec une approche géopolitique. On sent dans ses propos l'importance et le plaisir à enseigner de cette façon avec l'idée que sa démarche 
est porteuse de sens pour les élèves. C'est probablement celle qui insiste le plus dans l'entretien sur la formation des élèves, elle souhaite plus que tout leur donner des moyens de penser les événements.

On a vu que Jérôme évoque à plusieurs reprises, et longuement, la réussite de ses élèves à l'examen comme facteur déterminant de ses choix : «il faut mordicus ». Peut-être puise$\mathrm{t}$-il dans cet objectif le sentiment lui aussi d'une mission accomplie, dans laquelle il trouve la reconnaissance de ses compétences, de la part des élèves, de la part des parents, de la part peut-être aussi de ses collègues et de sa hiérarchie? On sent chez lui un plaisir à trouver et travailler avec ses élèves des repères qui pourraient faire la différence dans une copie d'examen.

Quant à Monique, l'idée de relever un « défi » que d'autres pensent impossible («Certains enseignent encore avec une chronologie dépassée ») semble être pour elle une motivation puissante, et la satisfaction d'y parvenir se perçoit dans l'enthousiasme et la détermination avec lesquels elle décrit ses choix et explique sa séquence. La satisfaction dont elle témoigne tient aussi à nos yeux au fait que ce qu'elle présente est le résultat d'une élaboration collective (fruit d'un travail de groupe, à ses yeux "validé par des collègues et par l'inspection ») qui semble lui procurer à la fois un sens à son métier et la reconnaissance d'un savoir-faire. Pour elle l'enseignant doit mettre ses éventuelles réticences de côté : «C'est très insécurisant: ne plus faire de la chronologie, comment dirais-je, classique, traditionnelle, dans laquelle les parents d'élèves se retrouvent, les élèves se sécurisent et les professeurs... (silence) tant pis pour eux ». Ce chapitre est également pour Monique un support idéal pour théâtraliser, ce qu'elle nous démontre très largement dans la façon de décrire son cours. On devine qu'elle reproduit pour partie devant nous, lors de l'entretien, les attitudes, les gestes, les répliques et la prosodie qu'elle utilise en classe devant les élèves.

\section{Conclusion}

\section{Logique des acteurs et conditions du changement}

Nous avons constaté, au cours de nos entretiens, des enseignants qui tentent, chacun à leurs manières, de répondre à la prescription, et dont les choix didactiques témoignent d'une évolution du rapport à la discipline. Mais nous avons aussi constaté que ces évolutions sont plus difficiles à mettre en œuvre lorsqu'elles supposent une remise en cause de leur rapport au métier, de leur rôle de professeur, dans la classe face à leurs élèves et au-delà, vis-à-vis des finalités de la discipline telles que chacun les perçoit. En effet, les préoccupations qu'ils expriment et qui président à leurs choix mettent en avant ce qui pour nous correspond à des finalités de la discipline (Madeleine et ses « repères »), ou plus généralement à des valeurs de l'école (Monique et sa crainte de l'iniquité, Jérôme et son souci de la réussite des élèves). Ces enseignants ne sont pas tout à fait ceux décrits par Tutiaux-Guillon (2008), pour qui « les enseignants s'attachent avant tout aux savoirs, à leur transmission et à leur appropriation par les élèves, et aux conditions qui permettent au cours de s'effectuer normalement ", ce qui les conduirait à en oublier les finalités de leur discipline. Si ces finalités ne semblent pas premières dans les préoccupations de ces enseignants elles n'en demeurent pas moins fortement présentes, comme chez leurs collègues québécois interrogés par Levasseur, Moisan et Cardin (2013). 

concernant « la résistance au changement ». Pour elle,

les enseignants font apparemment fi dans leurs pratiques des théories de l'apprentissage ou des travaux de didactique. Ils font comme si logique d'exposition et logique d'apprentissage se recouvraient... Or cette conception est à la fois cohérente avec l'organisation du temps scolaire et celle des programmes, et conciliable avec une lecture positiviste des savoirs. Changer l'enseignement serait alors changer non de programme, mais de système. souvent les savoirs factuels découpés en "tranches » (Audigier, 1995; Tutiaux-Guillon, 2008) (plus forcément de façon chronologique, mais comme autant d'unités indépendantes) qu'il ne reste plus qu'à traduire en autant de séances, dans un cadrage horaire qui ne laisse aucune marge de manœuvre. Pierre-Philippe Bugnard (2016) a bien mis en avant la contradiction entre ce découpage et la progression linéaire qui est celle des programmes d'une part, et la définition d'objectifs de compétences (ou, au lycée de capacités), d'autre part. De même, la contrainte temporelle fréquemment évoquée par les enseignants dès lors qu'il est question de «terminer» le programme peut être parfois perçue comme une forme de "résistance» à la prescription. Pourtant, elle pèse manifestement lourd, tant sur les choix didactiques que sur leur ressenti : "J'ai vécu ces années comme des courses contre la montre", nous dit Jérôme, tandis que Madeleine avoue "tricher » avec la prescription, et lorsque qu'on lui demande si cette contrainte de temps est propre au chapitre sur la guerre froide, elle déclare : "C'est sur tout le programme, elle est harassante cette première. » Nous avons d'ailleurs constaté que même la plus convaincue par la nouvelle démarche (Monique) ne peut s'en tirer qu'en externalisant une partie du travail de la classe, ce qui n'est certainement pas le but recherché. Pour ces enseignants, le cours magistral dialogué correspond certainement à leurs conceptions de l'enseignement-apprentissage, mais c'est aussi une solution acceptable pour finir le programme : au bout du compte tous savent que leur travail sera jugé sur ce critère (en particulier au lycée), plus que sur les méthodes qu'ils auront employées, ce qui explique aussi que cette pratique soit communément admise dans les classes à examen.

\section{Accompagner les enseignants}

Une autre condition à nos yeux nécessaire serait de réinterroger la forme du cours magistral dialogué, exercice de haut vol encore trop considéré comme un indicateur des compétences du professeur, y compris en formation initiale. Il s'agirait de travailler davantage sur les conceptions de l'enseignement et de l'apprentissage cohérentes avec la conception de l'histoire sous-jacente au programme (conceptualisation, démarche inductive, travail par compétences). Nos observations nous laissent penser que les enseignants ne sont pas fondamentalement opposés à l'évolution de leur enseignement. Les changements qu'ils opèrent dans leurs pratiques ne sont pourtant pas d'emblée intégrés au cours lui-même mais transférés "hors la classe", par exemple dans les dispositifs d'aide, comme si ces pratiques nouvelles, venaient en complément du cours, et n'étaient pas destinées à s'y substituer. On peut penser que c'est une façon pour eux de 
s'essayer à ces nouvelles méthodes sans remettre en cause trop brutalement l'équilibre fragile du cours. C'est l'une des pistes que nous nous proposons d'explorer dans une nouvelle opération de recherche actuellement en cours. Sur le plan méthodologique, et tout en reconduisant le dispositif des premiers entretiens, elle ajoute l'enregistrement filmique de séances de cours, des entretiens d'auto-confrontation ainsi que des entretiens croisés qui donnent l'occasion aux professeurs d'échanger entre eux sur leurs choix de conception et ce faisant, de faire émerger à leurs yeux de nouvelles démarches. Mieux comprendre la condition enseignante face à des changements de la prescription permet d'envisager des façons de les aider à trouver, dans leur expérience professionnelle et dans l'échange entre pairs, des compromis acceptables pour eux, pour les élèves, pour la discipline.

\section{BIBLIOGRAPHIE}

Audigier, F. (1995). Histoire et géographie : des savoirs scolaires en question entre les définitions officielles et les constructions des élèves. Spirale, 15, 61-89.

Audigier, F. (2005). Les enseignements d'histoire et de géographie aux prises avec la forme scolaire. Dans O. Maulini \& C. Montandon (dir), Les formes de l'éducation : variété et variations (p.103-122). Bruxelles : De Boeck Supérieur.

Boutonnet, V. (2015). Pratiques déclarées d'enseignants d'histoire au secondaire en lien avec leurs usages des ressources didactiques et l'exercice de la méthode historique. McGill Journal of Education/Revue des sciences de l'éducation de McGill, 50(2-3), 225-246.

Bugnard, P.-P., (2016). En histoire enseignée, l'évaluation des compétences se heurte à l'écueil de la progression. Dans M. A. Ethier \& E. Mottet (dir), Didactiques de l'histoire, de la géographie et de l'éducation à la citoyenneté : Recherches et pratiques (pp. 39-51). Bruxelles : De Boeck Supérieur.

De Cock, L. (2015). L'histoire scolaire, une matière indisciplinée. Annales. Histoire, Sciences Sociales, 70(1), 179-189.

Doussot, S. (2014). Continuité recherche, formation, enseignement en didactique de l'histoire. Problématisation historique et problématisation didactique. Revue française de pédagogie, 187, $55-70$.

Fontanabona, J. \& Thémines, J.-F. (2005). Innovation et histoire-géographie dans l'enseignement secondaire. Analyses didactiques. Lyon : INRP.

Goigoux, R. (2007). Un modèle d'analyse de l'activité des enseignants. Education \& Didactique, 3(1), 47-70.

Lantheaume, F. (2007). L'activité enseignante entre prescription et réel : ruses, petits bonheurs, souffrance ». Éducation et sociétés, 19(1), 67-81.

Lautier, N. (2003). Histoire enseignée, histoire appropriée. Quelques éléments spécifiques d'une didactique de l'histoire. Dans M.-C. Baquès, A. Bruter \& N. Tutiaux-Guillon, Pistes didactiques et chemins d'historiens (pp. 357-380), textes offerts à Henri Moniot. Paris : L'Harmattan. 
Legris, P. (2010). Les programmes d'histoire en France : la construction progressive d'une « citoyenneté

plurielle » (1980-2010). Histoire de l'éducation (126) DOI : 10.4000/histoire-education.2155

Legris, P. (2014). Qui écrit les programmes d'histoire? Grenoble : Presses universitaires de Grenoble.

LeVasseur, L., Moisan, S. \& Cardin, J. F. (2013). Les enseignants d'histoire et le programme d'Histoire et l'éducation à la citoyenneté : de la transmission de la mémoire à une citoyenneté « subjective » et ouverte. Phronesis, 2(2-3), 77-86.

Robert, A. \& Rogalski, J. (2002). Le système complexe et cohérent des pratiques des enseignants de mathématiques : une double approche. Revue canadienne de l'enseignement des sciences, des mathématiques et des technologies, 4(2), 505-528.

Theureau, J. (2010). « Les entretiens d'autoconfrontation et de remise en situation par les traces matérielles et le programme de recherche « cours d'action ». ». Revue d'anthropologie des connaissances, 2(4), 287-322.

Thémines, J.-F. \& Doussot, S. (2016). Les sciences sociales pour penser les acteurs dans les didactiques des sciences sociales (pp. 7-34). Dans Thémines, J.-F. \& Doussot, S. (dir), Acteurs et action en didactiques de l'histoire et de la géographie. Caen : PUC.

Tutiaux-Guillon, N. (2004). Les conceptions de l'apprentissage auxquelles se réfèrent les enseignants seraient-elles un facteur d'inertie disciplinaire? Journées d'études de didactiques de la géographie et de l'histoire (JED 2004, Caen). Repéré à http://ecehg.inrp.fr

Tutiaux-Guillon, N. (2009). L'histoire scolaire française entre deux modèles : contenus, pratiques et finalités. Raisons, Comparaisons, Educations, La Revue française d'éducation comparée, 4, 105-117.

Tutiaux-Guillon, N. (2008). Interpréter la stabilité d'une discipline scolaire : l'histoire-géographie dans le secondaire français. In François Audigier \& Nicole Tutiaux-Guillon (Ed.), Compétences et contenus les curriculums en question (pp. 117-146). Bruxelles : De Boeck.

\section{NOTES}

1. Afin de ne pas alourdir le texte, nous avons opté pour l'utilisation du masculin. Cette forme désigne donc ici des hommes et des femmes.

2. Nous tenons à remercier les enseignants et enseignantes qui ont accepté de nous accorder de leur temps et de nous montrer leur travail.

3. Le texte de Doussot et Thémines (2016) introduction aux actes du colloque international tenu à Caen en 2014, présente une intéressante synthèse de l'histoire et des enjeux de l'approche par les acteurs en didactique des sciences sociales.

4. Sur la "fabrique scolaire de l'histoire", voir l'ouvrage de L. De Cock et E. Picard (2010), ou l'article de L. De Cock (2015) : L'histoire scolaire, une matière indisciplinée. Annales. Histoire, sciences sociales, 179-189.

5. Depuis les années 60, les programmes effectuent des sauts dans le temps et accordent moins d'importance aux dates et aux événements. Le programme de 2002 se place dans cette évolution, en focalisant sur les «traits majeurs » des modèles soviétique et américain » et les «lignes de force » du conflit Est-Ouest.

6. Berlin jusqu'en 89 avant de revenir sur la crise de Cuba, le chapitre sur les totalitarismes après celui sur la seconde guerre mondiale, par exemple.

7. «Le fait de s'attacher à des objets précis et significatifs ne signifie pas que l'on puisse généraliser leurs caractères propres et construire un savoir historique général qui serait déduit de leur singularité. Il s'agit 
de mettre l'accent sur la dimension heuristique de ces objets, c'est-à-dire plus précisément en montrant en quoi ils sont porteurs de sens. Ils n'impliquent pas une généralisation mécanique a posteriori mais posent au contraire comme préalable la justification de leur choix.».

http://cache.media.eduscol.education.fr/file/lycee/69/6/

LyceeGT_Ressources_Hist_1_01_Demarches_184696.pdf

LyceeGT_Ressources_Hist_1_01_Demarches_184696.pdf

9. http://cache.media.eduscol.education.fr/file/

HG_series_ES_et_L_mise_a_jour_1ere/55/5/04_Hist_Th2_Q2_De_la_guerre_froide_a_de_NC_VF_458555.pdf

10. http://cache.media.eduscol.education.fr/file/lycee/69/6/

LyceeGT_Ressources_Hist_1_01_Demarches_184696.pdf

11. En réalité ces dimensions sont présentes, de façon plus ou moins prégnante, chez les trois enseignants. Nous présentons ici la dimension qui est le plus ressortie de chaque entretien.

\section{RÉSUMÉS}

Comment les enseignants d'histoire géographie composent avec les évolutions de la prescription? Trois portraits permettent de mettre en évidence la façon dont ils redéfinissent la tâche (Leplat, 1980) chacun à leur manière pour concevoir leur enseignement de la guerre froide. L'analyse de leur activité de conception vise à faire état des tensions inhérentes à leurs choix et aux compromis trouvés face à de multiples déterminants et adressages (Goigoux, 2007). Ces compromis sont mis en perspective avec les travaux de didactique (Audigier, 1995 ; Lautier, 2003 ; Tutiaux-Guillon, 2004, 2008, 2009) pour développer une réflexion sur les conditions d'évolution du modèle didactique, des conceptions et des pratiques enseignantes.

How do history and geography teachers deal with changes in prescription? Three portraits are described to highlight how they redefine, each in their own way, the task (Leplat, 1980) of designing their teaching of the Cold War. The aim of the analysis of their activity is to describe tensions linked to their choices and compromises in front of numerous referents and determinants of the activity (Goigoux, 2007). These compromises are put into perspective with didactic researches (Audigier, 1995; Lautier, 2003; Tutiaux-Guillon, 2004, 2008, 2009) to develop a new way of thinking the conditions of development of teaching practices.

\section{INDEX}

Keywords : teaching, history, prescription, didactics, activity

Mots-clés : enseignement, histoire, prescription, didactique, activité

\section{AUTEURS}

\section{BRIGITTE MORAND}

Université, Clermont Auvergne, ESPE Clermont Auvergne, Laboratoire ACTé 


\section{GUILLAUME SERRES}

Université, Clermont Auvergne, ESPE Clermont Auvergne, Laboratoire ACTé 